\title{
DEMANDA TRACTIVA EN FUNCIÓN DE LA RESISTENCIA MECÁNICA DEL SUELO A LA PENETRACIÓN
}

\author{
PEDRO H. M. BORGES ${ }^{1}$, ALOÍSIO BIANCHINI ${ }^{2}$, JOÃO C. S. MAIA ${ }^{3}$, \\ HAROLDO C. FERNÁNDES ${ }^{4}$, ZAÍRA M. S. H. MENDOZA ${ }^{5}$
}

\begin{abstract}
RESUMO: No presente estudo, foram elaborados modelos empíricos para determinar a força de tração demandada por arados de discos, escarificadores e semeadoras adubadoras, em função da resistência mecânica do solo à penetração. A média da referida resistência, determinou-se até as profundidades de 25; 35 e $15 \mathrm{~cm}$, em correspondência com as regulagens do arado de discos, escarificador e semeadora adubadora. O ajuste foi verificado de acordo com o coeficiente de determinação, gráficos de dispersão, análise residual e teste t (Student). Com base nessas análises, confirmou-se a normalidade dos resíduos, e foram estabelecidos intervalos de confiança com 95\% de probabilidade. Os valores estimados e obtidos experimentalmente não discreparam significativamente. Concluiu-se que as funções exponenciais associadas apresentaram adequada precisão e confiabilidade para predizer a força de tração, considerando-se a resistência mecânica do solo à penetração e que os modelos podem ser uma ferramenta útil para o planejamento e gestão de operações agrícolas mecanizadas em solos com textura similar às desta pesquisa.
\end{abstract}

PALAVRAS-CHAVE: desempenho de máquinas, modelos empíricos, modelos não lineares.

\section{TRACTIVE DEMAND AS FUNCTION OF SOIL MECHANICAL RESISTANCE TO PENETRATION}

\begin{abstract}
In this study, empirical models were developed to determine the tensile force demanded by disc plows, harrows and planters fertilizers, as function of soil mechanical resistance to penetration. The mean of the referred resistance was determined until 25, 35 and $15 \mathrm{~cm}$ depths, according to the regulations for the disc plow, springs harrow and fertilizer seeder. The adjustment was verified based on the coefficient of determination, dispersion graphics, residual analysis and $t$ test (Student). It was confirm the normality of the residuals and to establish intervals of confidence with $95 \%$ of probability. The estimated and experimentally obtained values did not differ significantly. It was concluded that the associated exponential functions presented appropriate precision and reliability to predict the traction force being considered the soil mechanical resistance to penetration and that the models can be a useful tool for planning and management of mechanized farming operations in soils with texture similar to those of this research.
\end{abstract}

KEYWORDS: machine performance; empirical models; nonlinear models.

\section{INTRODUCCIÓN}

Para implantar una cultura agrícola, se puede preparar el terreno convencionalmente o adoptando métodos conservacionistas. En el preparo convencional existe elevada movilización del suelo causada por arados y gradas. Por otro lado, los métodos conservacionistas tienen por finalidad disminuir el número de operaciones, como es el sistema reducido utilizándose un escarificador o aún eliminar totalmente esas actividades como es en el plantío directo, realizado solamente con una sembradora abonadora apropiada. Sin embargo, independientemente del método de preparo, para

\footnotetext{
${ }^{1}$ Ing. Agrícola, Prof. Doctor, Departamento de Suelos e Ingeniería Rural, UFMT/Cuiabá-MT, Teléfono: 6536158433 , pborges@ufmt.br

2 Ing. Agrónomo, Prof. Associado, Departamento de Suelos e Ingeniería Rural, UFMT/Cuiabá-MT, bianchi@ufmt.br

3 Ing. Agrónomo, Prof. Asociado, Departamento de Suelos e Ingeniería Rural, UFMT/Cuiabá-MT, jotace@terra.com.br

${ }^{4}$ Ing. Agrícola, Prof. Asociado, Departamento de Ingeniería Agrícola, UFV/Viçosa-MG, haroldo@ufv.br

${ }^{5}$ Ing $^{\text {a }}$ Forestal, Prof ${ }^{\text {a }}$. Adjunta, Departamento de Ingeniería Forestal, UFMT/Cuiabá-MT, zaira@ufmt.br

Recebido pelo Conselho Editorial em: 17-10-2012

Aprovado pelo Conselho Editorial em: 25-11-2013
} 
elevar los rendimientos de las culturas es necesario seleccionar y dimensionar correctamente los tractores de acuerdo con las máquinas e implementos correspondientes.

Establecer una relación adecuada entre el tractor y el implemento o máquina, significa formar racionalmente un conjunto mecanizado, lo que implica en disminuir los costos de producción y los impactos ambientales como por ejemplo excesiva erosión o compactación de los suelos. La formación racional de conjuntos mecanizados requiere el conocimiento de la demanda tractiva en la operación. En este sentido, ASAE (2003) desarrolló modelos empíricos que determinan la referida fuerza con base en parámetros de las máquinas e implementos y del suelo. No obstante, esos modelos son muy limitados, pues no presentan variables para considerar el estado actual del terreno en relación al contenido de agua, grado de compactación y tipo de cobertura, entre otros.

Debemos destacar que los modelos propuestos por la ASAE (2003) fueron establecidos en condiciones experimentales, probablemente, muy distintas a las presentes en los suelos brasileños, llevando a la determinación de valores de fuerza significativamente diferentes. Este aspecto fue abordado por SANTOS (2010), quien comprobó que esos modelos subestimaron la fuerza requerida por escarificadores y sembradoras abonadoras. MODOLO et al. (2004, 2005), SILVEIRA et al. (2005 a, b), FURLANI et al. (2007), MACHADO et al. (2007), FERNANDES \& GAMERO (2010), SILVEIRA et al. (2011) estudiaron la fuerza de tracción requerida por diversos equipos agrícolas. No obstante, los resultados obtenidos por los referidos autores no pueden ser extrapolados con seguridad para condiciones distintas de las citadas en sus experimentos.

Una variable muy utilizada para representar el estado actual del terreno es la resistencia mecánica media del suelo a la penetración para determinada profundidad. MOLIN et al. (2006) verificaron que la mencionada resistencia es influenciada principalmente por el contenido de agua y la densidad del suelo, siendo eficiente para indicar su compactación. También, BEUTLER \& CENTURION (2004); BEUTLER et al. (2006); PÉREZ (2006); ACOSTA (2008); BONNIN et al. (2010) emplearon esa variable como principal indicativo para evaluar la compactación del terreno. Sin embargo, en esos estudios no se desarrollaron dependencias funcionales para relacionar la fuerza de tracción de implementos y máquinas agrícolas, considerándose el estado de compactación del suelo. Esta investigación se fundamentó en la hipótesis de que la referida fuerza varía con la resistencia del terreno, teniendo como objetivo estimarla para arado de discos, escarificadores y sembradoras abonadoras, en función de la resistencia mecánica media del suelo a la penetración, de acuerdo con la profundidad de trabajo.

\section{MATERIAL Y MÉTODOS}

El experimento fue conducido durante dos años consecutivos, en un área perteneciente a la Universidad Federal de Viçosa, situada en la ciudad de Viçosa-MG, definida por las coordenadas geográficas $20^{\circ} 45^{\prime} 14$ ” de latitud Sur y $42^{\circ} 52^{\prime} 53^{\prime \prime}$ de longitud Oeste y altitud media de $650 \mathrm{~m}$. En la región predomina el relevo montañoso (aproximadamente el 85\%). El terreno fue sometido a la siembra directa durante los últimos cinco años con los cultivos de maíz y frijol de forma alternada.

La temperatura anual media varía entre $14,0^{\circ} \mathrm{C}$ y $26,1^{\circ} \mathrm{C}$. El clima fue clasificado como Cwa, templado húmedo con veranos calientes e inviernos secos (KÖEPPEN \& GEIGER, 1928) y el suelo como Latosuelo rojo-amarillo de textura franco-arcillosa (EMBRAPA, 1999). Las características climáticas de la región permiten definir en el año tres épocas: húmeda (enero, febrero, noviembre y diciembre), intermedia (marzo, abril, septiembre y octubre) y seca (mayo, junio, julio y agosto). Los respectivos contenidos de agua (base volumétrica) en el suelo varían de 35 a 45\%, de 25 a 35\% y de 15 a 25\%, para los períodos húmedo, intermedio y seco. En la Tabla 1 se presentan los valores medios de las variables utilizadas para caracterizar el área experimental. 
TABLA 1. Valores medios de las variables utilizadas para caracterizar el área experimental. Mean values of the variables used to characterize the experimental area.

\begin{tabular}{ccc}
\hline \multicolumn{2}{c}{ Variable $\mathrm{y}$ unidad } & Valor medio \\
\hline Masa seca de la cobertura vegetal $\left(\mathrm{t} \mathrm{ha}^{-1}\right)$ & & 5,59 \\
\hline Densidad del suelo $\left(\mathrm{g} \mathrm{cm}^{-3}\right)$ & & 1,53 \\
\hline \multirow{2}{*}{ Contenido de agua en el suelo } & Período húmedo & 42,78 \\
$(\% \mathrm{bv})$ & Período intermedio & 28,62 \\
& Período seco & 19,97 \\
\hline \multirow{2}{*}{ Porosidad del suelo } & Macro porosidad & 12,83 \\
$(\%)$ & Micro porosidad & 38,64 \\
Textura del suelo & Porosidad total & 51,47 \\
$(\%)$ & Arcilla & 48,06 \\
& Limo & 26,93 \\
& Arena gruesa & 7,19 \\
\end{tabular}

Para realizar esta investigación se utilizaron dos tractores de ruedas con tracción delantera auxiliar (4x2 TDA). El primero, modelo MF 265, fabricado por la Massey Ferguson ${ }^{*}$, con potencia nominal a $2200 \mathrm{rpm}$ de 47,8 kW y masa con lastre de $3700 \mathrm{Kg}$. El segundo, modelo 7630, fabricado por la Valtra*, con potencia nominal a $2200 \mathrm{rpm}$ de 75,8 kW y masa con lastre de $6196 \mathrm{Kg}$. Como implementos y máquinas, montados en el sistema de levante hidráulico del tractor, se emplearon un arado reversible con 3 discos de 29", un escarificador de muelles constituido por 6 astas parabólicas de ancho $30 \mathrm{~mm}$ con punteras de tipo común sin alas y una sembradora-abonadora para plantío directo, modelo PC 2123, fabricada por la SEED MAX*, compuesta por tres unidades pantográficas separadas a $450 \mathrm{~mm}$ con discos de corte liso de diámetro $406 \mathrm{~mm}$, dosificador de semillas y fertilizantes, surcadores de discos dobles y ruedas compactadoras lisas en forma de "V".

También, fueron necesarios otros equipos y herramientas computacionales de soporte como el penetrómetro electrónico, modelo PNT 2000*, fabricado por la DLG Automación Industrial Ltda*, la celda de carga, marca SODMEX ${ }^{*}$ y modelo $\mathrm{N} 400$, con sensibilidad de 2,156 $\mathrm{mV} \mathrm{V}^{-1}$ y escala nominal de $50 \mathrm{kN}$, computadora portátil, modelo ASPIRE 5920, fabricado pela ACER ${ }^{*}$, con sistema operacional WINDOWS XP, planilla electrónica EXCEL de la empresa Microsoft, programa para ajuste de curvas CurveExpert 1.4 y programa estadístico STATISTICA 8.0.

El tractor MF 265 se destinó para el montaje de los equipos agrícolas en su sistema de levante hidráulico, mientras el tractor Valtra 7630 para remolcar el conjunto mecanizado y entre los dos se acopló la celda de carga. El arado, el escarificador y la sembradora abonadora fueron regulados para las profundidades de 25, 35 y $15 \mathrm{~cm}$, respectivamente. Durante las lecturas de fuerza de tracción en la celda de carga, el tractor remolcador operó en segunda marcha reducida con el motor a $1700 \mathrm{rpm}$, siendo que inicialmente los implementos estaban suspendidos y después en la posición de trabajo correspondiente a las regulaciones de profundidad. Luego, la fuerza de tracción requerida por el implemento fue determinada por la diferencia entre las dos lecturas.

Para planificar la colecta de los datos, el terreno de forma rectangular con $140 \mathrm{~m}$ de largo y $110 \mathrm{~m}$ de ancho fue dividido en dos partes, siendo una para cada año. En cada mitad del terreno se definieron 9 áreas, destinadas a los 3 equipos agrícolas (arado, escarificador y sembradora abonadora) y a los 3 períodos del año. Finalmente en esas áreas fueron demarcadas 20 unidades experimentales con $5 \mathrm{~m}$ de largo y $3 \mathrm{~m}$ de ancho, correspondiendo 10 parcelas para cada mes y equipo agrícola en todo el trabajo.

En las parcelas experimentales se realizaron inicialmente 5 lecturas aleatorias de la resistencia mecánica media del suelo a la penetración para la profundidad correspondiente al implemento o máquina y 5 de la fuerza de tracción en la barra, calculándose posteriormente la media entre los dos años de investigación. Luego, los meses se representaron por 5 observaciones, totalizando 60 pares 
de valores para cada implemento o máquina. Los modelos empíricos para describir analíticamente la fuerza requerida para la tracción de los equipos agrícolas, en función de la referida resistencia media se elaboraron con ayuda de los módulos disponibles en los programas computacionales. La calidad del ajuste se evaluó con base en el coeficiente de determinación, gráficos de dispersión, análisis residual y Test t, conforme sugerido por MONTGOMERY et al. (2004), LEVINE et al. (2005) y WEISBERG (2005).

* La citación de marcas no significa recomendación de los autores, sino la caracterización de los equipos utilizados en esta investigación.

\section{RESULTADOS Y DISCUSIÓN}

Dentro de las diversas expresiones matemáticas que podían representar los valores de fuerza de tracción, considerándose el índice de cono, se optó por la función exponencial asociada para todos los equipos agrícolas. Ese modelo empírico tiene como características el inicio en el origen del sistema de coordenadas, el aumento de la fuerza en la barra de tracción con el incremento del índice de cono y la definición de un valor asintótico para a variable dependiente. La tendencia de los datos experimentales concuerdan adecuadamente con las características del referido modelo, cuya forma general es dada por:

$$
\mathrm{Ftr}=\mathrm{A}\left(1-\mathrm{e}^{-\mathrm{k} \mathrm{IC}_{\mathrm{p}}}\right)
$$

en que,

$\mathrm{F}_{\mathrm{tr}}$ - Fuerza de tracción requerida, cuja unidad depende del implemento, así se tiene:

- específica por área trabajada para el arado $\left(\mathrm{N} \mathrm{cm}^{-2}\right)$ y

- por línea de trabajo para el escarificador y sembradora $\left(\mathrm{kN}_{\text {asta }}{ }^{-1} \mathrm{o}\right.$ línea $\left.{ }^{-1}\right)$;

$\mathrm{IC}_{\mathrm{p}}$ - Índice de cono a la profundidad alcanzada por el equipo agrícola (MPa);

A - Parámetro del modelo que representa el valor asintótico de la fuerza requerida, luego:

- específica por área trabajada para el arado $\left(\mathrm{N} \mathrm{cm}^{-2}\right)$ y

- por línea de trabajo para el escarificador y sembradora $\left(\mathrm{kN}_{\text {asta }}{ }^{-1} \mathrm{o}\right.$ línea $\left.{ }^{-1}\right)$;

k - Parámetro del modelo que indica la velocidad en el aumento de fuerza (adimensional).

Los valores, unidades y estadísticas de los parámetros para las ecuaciones ajustadas, así como los coeficientes de determinación $\left(\mathrm{R}^{2}\right)$ correspondientes a cada equipo agrícola se encuentran en la Tabla 2. A pesar de los elevados valores del coeficiente de determinación, este no es el único índice para evaluar el ajuste, pues se trata de un modelo no lineal. Los bajos valores de probabilidad indican más del 99\% de confianza para aceptar los parámetros de los modelos obtenidos (Tabla 2).

TABLA 2. Valores, unidades y estadísticas de los parámetros del modelo ajustado, bien como los respectivos coeficientes de determinación $\left(\mathrm{R}^{2}\right)$ para cada equipo agrícola.Values, units and statistics of the parameters of the adjusted models, as well as the respective determination coefficient $\left(R^{2}\right)$ for each farm equipment.

\begin{tabular}{ccccccc}
\hline \multirow{2}{*}{ Tipo de información } & \multicolumn{2}{c}{ Arado de discos } & \multicolumn{2}{c}{ Escarificador de muelles } & \multicolumn{2}{c}{ Sembradora abonadora } \\
\cline { 2 - 7 } & $\mathrm{A}$ & $\mathrm{k}$ & $\mathrm{A}$ & $\mathrm{k}$ & $\mathrm{A}$ & $\mathrm{k}$ \\
\hline Valor & 9,6142 & 0,6164 & 5,6612 & 0,7169 & 4,5828 & 0,6992 \\
Unidad & $\mathrm{N} \mathrm{cm}^{-2}$ & - & $\mathrm{kN} \mathrm{asta}^{-1}$ & - & $\mathrm{kN} \mathrm{línea}^{-1}$ & - \\
Desviación estándar & 0,9289 & 0,1332 & 0,7039 & 0,0956 & 0,8908 & 0,0720 \\
Valor t & 10,3501 & 4,6276 & 8,0409 & 7,4999 & 5,1448 & 9,2000 \\
Valor de probabilidad & 0,0023 & 0,0004 & 0,0017 & 0,0009 & 0,0038 & 0,0012 \\
IC $_{95 \%}$ & $\pm 1,8587$ & $\pm 0,2665$ & $\pm 1,4085$ & $\pm 0,1913$ & $\pm 1,7825$ & $\pm 0,1521$ \\
$\mathrm{R}^{2}$ & \multicolumn{2}{c}{0,9817} & \multicolumn{2}{c}{0,9725} & 0,9411 \\
\hline
\end{tabular}

Obs. IC $_{95 \%}$ : Intervalo de confianza para un 95\% de probabilidad. A, k: Parámetros del modelo ajustado. 
En las Figuras 1, 2 y 3 se pueden observar los gráficos de dispersión con los datos experimentales, la curva de la ecuación ajustada para la fuerza requerida en la barra de tracción, en función del índice de cono correspondiente a los implementos estudiados y los límites del intervalo de confianza para $95 \%$ de probabilidad. Se puede constatar un aumento de la referida fuerza con el incremento del índice de cono, siendo más acentuado en el inicio (Figuras 1, 2 y 3). De acuerdo con esas Figuras, la curva ajustada acompaña adecuadamente la tendencia presentada por los valores experimentales y todos estos variaron dentro del intervalo de confianza establecido.

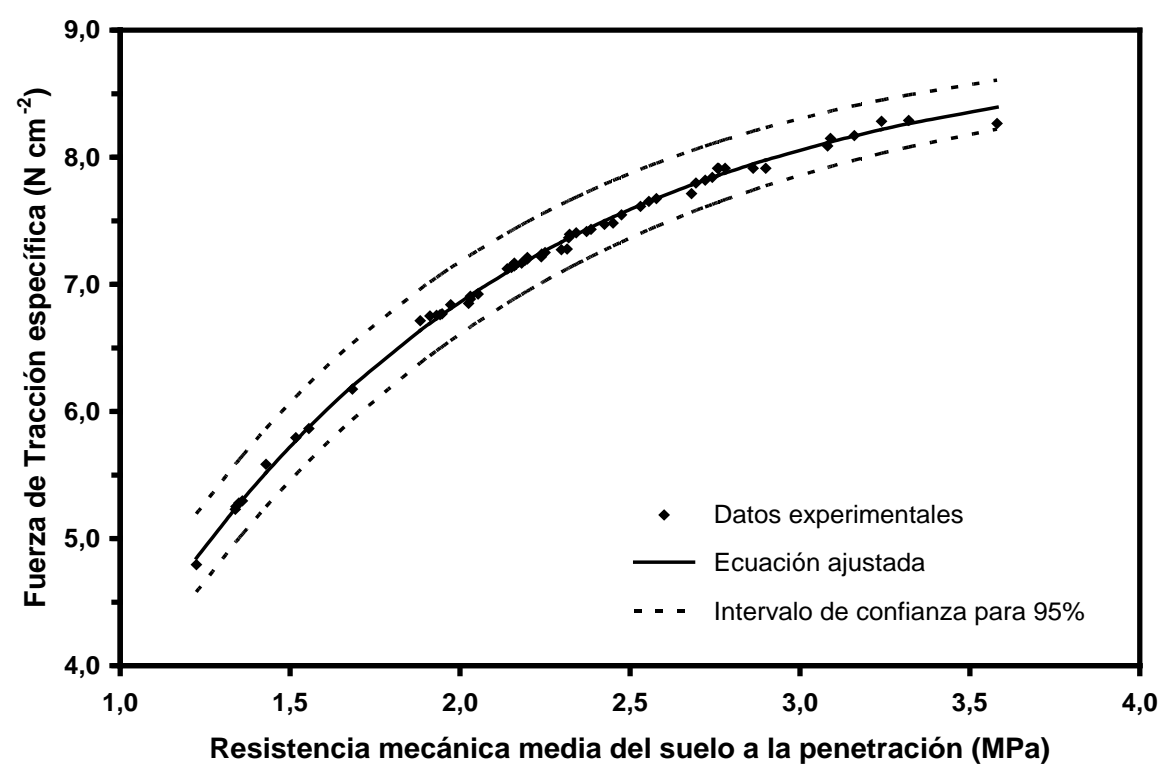

FIGURA 1. Datos experimentales y curva de la ecuación ajustada para el arado de discos. Experimental data and curve of the adjusted equation for the disk plow.

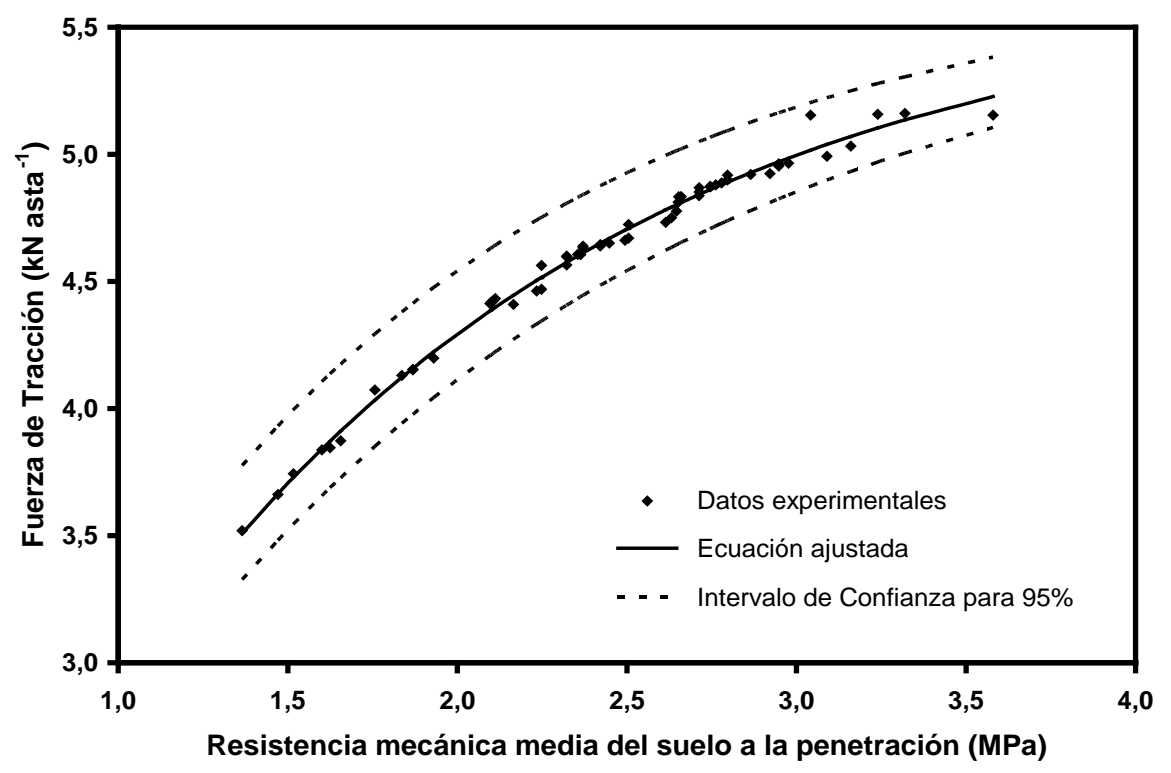

FIGURA 2. Datos experimentales y curva de la ecuación ajustada para el escarificador de muelles. Experimental data and curve of the adjusted equation for the springs harrow. 


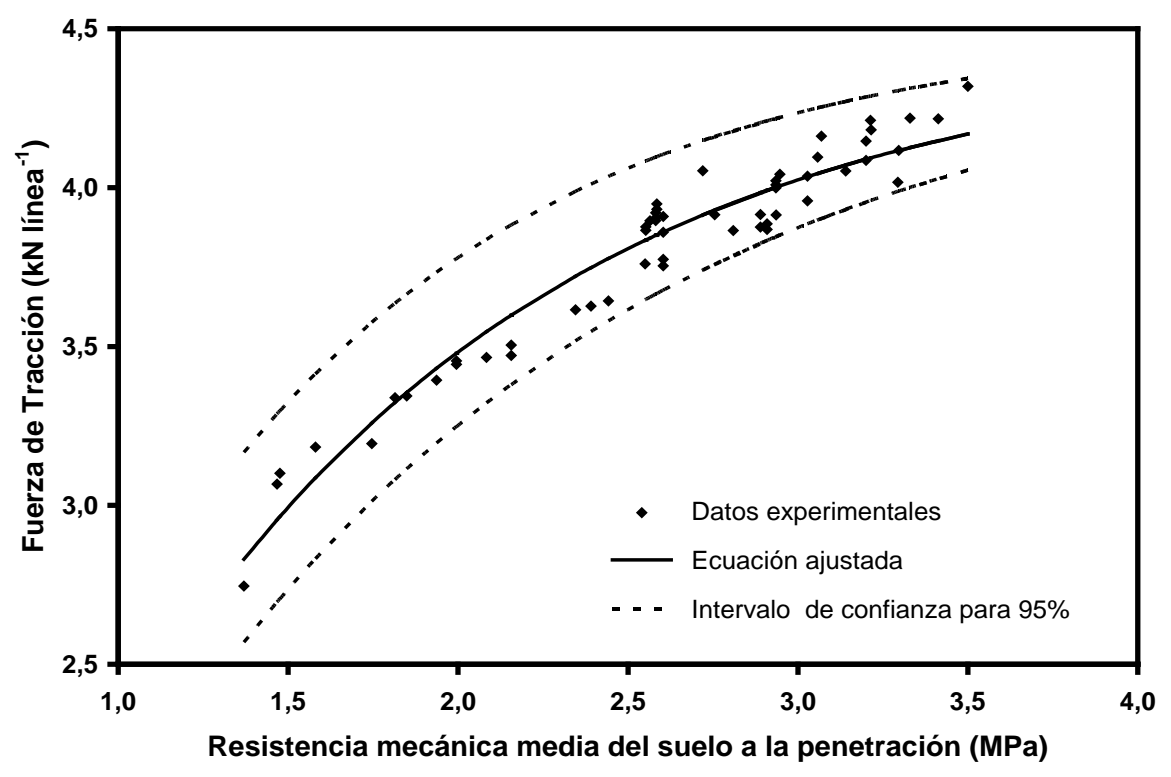

FIGURA 3. Datos experimentales y curva de la ecuación ajustada para la sembradora abonadora. Experimental data and curve of the adjusted equation for the fertilizer seeder.

Conforme las Figuras 1, 2 y 3, la resistencia mecánica media del suelo a la penetración varió entre 1,35 y 3,50 MPa. El límite inferior en torno de 1,35 MPa se encuentra próximo al estimado por MOLIN et al. (2006). Esos autores evaluando la referida resistencia en un suelo con histórico de manejo, contenido de arcilla y contenido de agua semejantes a las de nuestra investigación obtuvieron 1,50 MPa como mínimo. No obstante, el valor máximo presentado por los referidos autores de 3,06 MPa es inferior al de este estudio. También, el límite superior de este trabajo está próximo al determinado por ASSIS \& LANÇAS (2005) de 3,39 $\mathrm{MPa}$ en condiciones experimentales similares y fue superior a los estimados por ASSIS et al. (2009) de 2,98 MPa, CARVALHO et al. (2006) de 2,8 MPa y CUNHA et al. (2009) de 2,6 MPa. Esas diferencias pueden ser originadas por la mayor amplitud del contenido de agua considerado en esta investigación, lo que representa un margen de seguridad para los modelos.

Los limites para la resistencia mecánica media del suelo a la penetración de este trabajo fueron superiores a los determinados por VENIALGO et al. (2004), que variaron entre 0,61 y 2,67 MPa. Esas discrepancias se deben, probablemente, a la rotación de culturas en ese estudio, incluyendo el algodón que posee un sistema radicular más profundo, el cual podría favorecer la disminución de la resistencia del suelo a la penetración en etapas posteriores. El intervalo de la resistencia mecánica establecido en nuestra investigación incluye los rangos de valores obtenidos por BEUTLER et al. (2006), WEIRICH et al. (2006), respectivamente, de 2,0 a 3,0 MPa y de 2,0 a 2,2 MPa. La mayor amplitud de nuestro intervalo de valores les confiere adecuada confiabilidad a los modelos propuestos para estimar la fuerza de tracción en situaciones extremas relativas al tipo de suelo y contenido de agua.

De acuerdo con los límites de confianza de la Figura 1, podemos afirmar con un nivel de significancia de $5 \%$ que la fuerza de tracción específica requerida por el arado de discos varió aproximadamente de 4,5 a 8,5 $\mathrm{N} \mathrm{cm}^{-2}$. Ese intervalo comprende los valores obtenidos por MELLO \& MAGALHÃES (1995) entre 6,37 y 7,22 $\mathrm{N} \mathrm{cm}^{-2}$, bien como la media determinada por FERNANDES \& GAMERO (2010) de 7,16 N cm${ }^{-2}$. Debemos destacar que en la mayoría de los trabajos consultados, se constataron valores de resistencia mecánica del suelo a la penetración entre 2 y $3 \mathrm{MPa}$. En ese intervalo la fuerza de tracción específica varió de 6,5 a 7,5 $\mathrm{N} \mathrm{cm}$ (Figura 1), valores próximos a los estimados en los estudios comentados. Esos resultados indican adecuada predicción del modelo. 
Conforme la Figura 2, la fuerza de tracción por asta demandada por el escarificador de muelles, varió en una faja de valores entre 3,4 y 5,4 $\mathrm{kN}$ asta $^{-1}$ con el $95 \%$ de probabilidad. Ese rango se encuentra dentro del intervalo sugerido por ASAE (2003) de 3,08 a 3,6 kN asta ${ }^{-1}$ y el determinado por SANTOS (2010) entre 3,8 y 4,7 $\mathrm{kN}$ asta $^{-1}$. Sin embargo, SALVADOR et al. (2008) estimaron valores de 2,7 a 4,2 $\mathrm{kN}_{\text {asta }}{ }^{-1}$. Esas discrepancias pueden ser debidas al preparo utilizado por esos autores, antes de realizar la escarificación, lo que contribuyó para disminuir la resistencia del suelo a la penetración. De todas formas, aunque las ecuaciones sobreestimen la fuerza de tracción para bajos valores de la mencionada resistencia, esto serviría como margen de seguridad, confiriéndole confiabilidad al modelo empírico propuesto.

Considerándose los limites de confianza para un 95\% de probabilidad de la Figura 3 se deduce que la fuerza de tracción por línea de siembra fluctuó aproximadamente entre 2,6 y 4,25 kN línea $^{-1}$. Ese intervalo supera la faja de valores establecida por ASAE (1996) de 1,1 a 2 kN línea ${ }^{-1}$, los obtenidos por FURLANI et al. (2004) de 2,15 a 2,42 $\mathrm{kN}$ línea $^{-1}$ y por MERCANTE et al. (2005) entre 2,1 y 2,5 kN línea ${ }^{-1}$, así como también el valor medio determinado por MODOLO et al. (2005) de 2,1 kN línea ${ }^{-1}$. No obstante, el intervalo definido en este estudio contiene el rango indicado por LEVIEN et al. (1999) entre 3,24 y 3,64 $\mathrm{kN}$ línea $^{-1}$ y el valor medio estimado por FURLANI et al. (2007) de 2,97 $\mathrm{kN}$ línea $^{-1}$. La mayor amplitud de valores observada en el presente experimento puede ser debida a las condiciones extremas del contenido de agua, aspecto determinante en las fluctuaciones de la resistencia del suelo a la penetración.

Por otro lado, el límite inferior de 2,6 kN línea-1 del presente trabajo pertenece al intervalo verificado por ALMEIDA et al. (2010), cuyos valores oscilaron entre 2,26 y 2,51 kN línea ${ }^{-1}$. También, el límite superior correspondiente a esta investigación de 4,25 kN línea ${ }^{-1}$ se encuentra dentro del rango obtenido por SILVEIRA et al. (2005 b) de 3,90 a 4,53 kN línea ${ }^{-1}$, respectivamente, para la menor y mayor profundidad de trabajo. Estas discrepancias pueden estar influenciadas por los mecanismos para abrir y cerrar los surcos, cortar la paja, distribuir semillas y fertilizantes, bien como estar relacionadas con el tipo y estado de la cobertura del terreno, los cuales son factores que interfieren en la resistencia del suelo. A pesar de las diferencias se considera el modelo confiable.

Para verificar el ajuste de los modelos empíricos para cada implemento, se elaboraron los diagramas de dispersión con los valores de la fuerza en la barra de tracción observada, en función de los estimados (Figura 4). En esa Figura, también se incluyó la bisectriz del cuadrante con la finalidad de identificar la relación entre los valores teóricos y reales. Se puede constatar que la mayoría de los puntos están distribuidos uniformemente encima y debajo de la bisectriz para los tres equipos agrícolas (Figura 4). También, esos puntos se encuentran próximos de la referida recta, indicando que los modelos obtenidos pueden ser apropiados para determinar la fuerza en la barra de tracción, considerándose la resistencia mecánica media del suelo a la penetración.

La premisa de normalidad de las diferencias entre los valores teóricos y reales para cada equipo agrícola es un aspecto muy importante para definir criterios sobre el ajuste de los modelos empíricos elaborados y se puede evaluar con base en los histogramas de residuos presentados en la Figura 5. Conforme esa Figura se puede deducir que las diferencias mencionadas se distribuyeron con adecuada simetría y muy próximas de la curva normal, es decir, la mayoría de pequeños residuos concentrados en el centro y una menor cantidad en los extremos.

Aunque la distribución de los residuos se aproximó a la curva normal, el análisis de los histogramas se considera un procedimiento subjetivo. Así, se optó por comprobar analíticamente la normalidad mediante las pruebas de Kolmogorov-Smirnov, Lilliefors y Shapiro-Wilk. Los resultados correspondientes a esas pruebas se presentan en la Tabla 3. Los valores de probabilidad obtenidos muy superiores al 5\% permiten afirmar que realmente la premisa de normalidad de los residuos para los tres implementos fue satisfecha. 

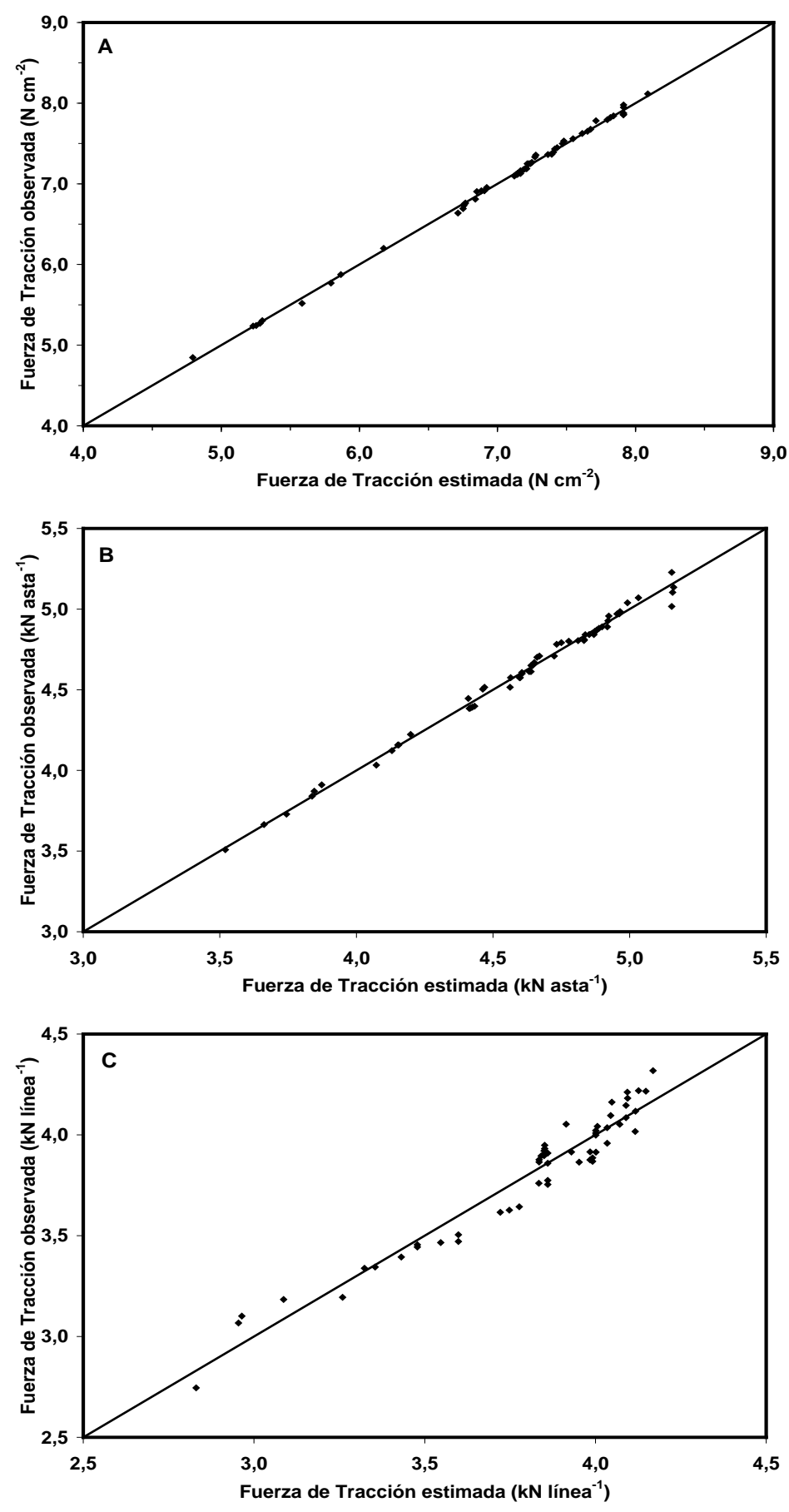

FIGURA 4. Bisectriz y valores observados de la fuerza en la barra de tracción, en función de los estimados: a) arado de discos; b) escarificador de muelles; c) sembradora abonadora.Bisector and observed values of the force on the drawbar, as function of the estimates: a) disk plow; b) springs harrow; c) fertilizer seeder. 

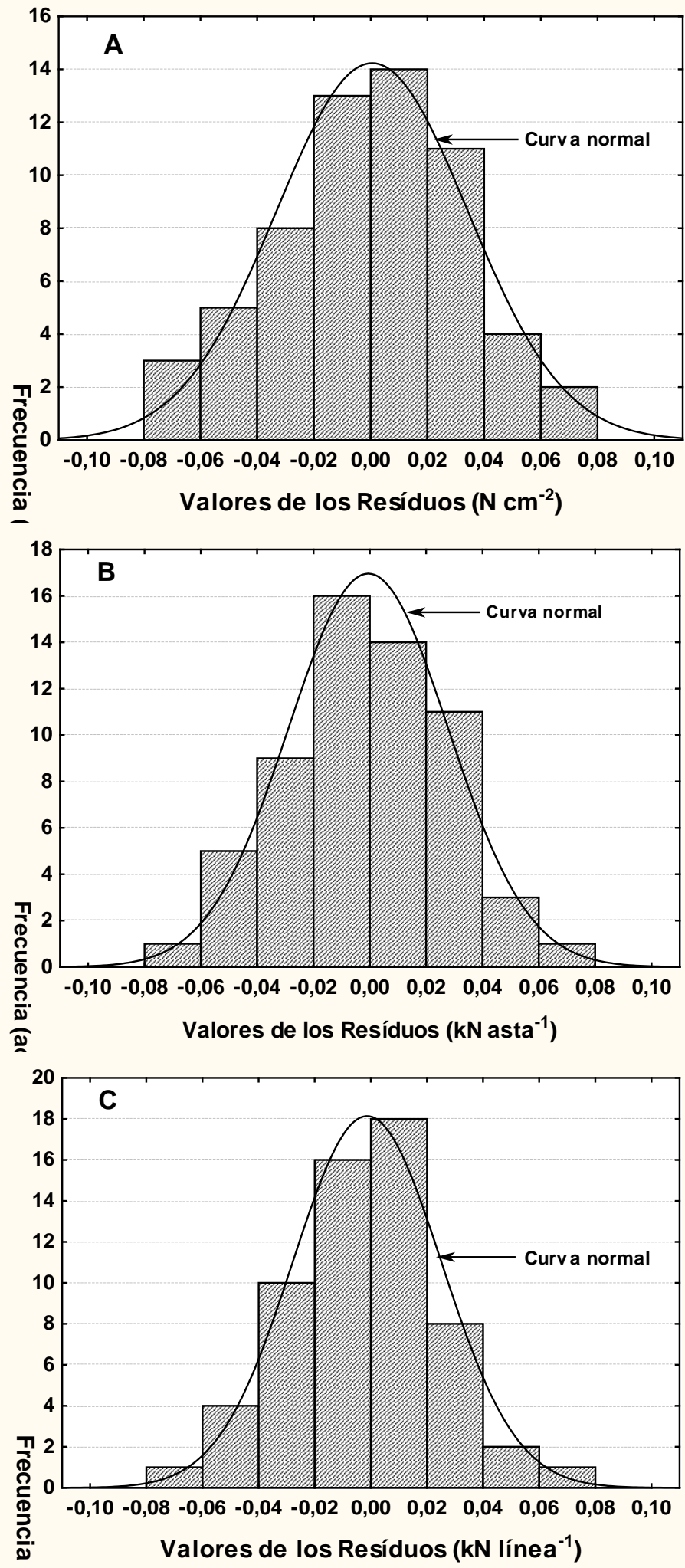

FIGURA 5. Histograma de los residuos: a) arado de discos; b) escarificador de muelles; c) sembradora abonadora. Histogram of residues: a) disk plow; b) springs harrow; c) fertilizer seeder.

TABLA 3. Resultados de las pruebas de normalidad para los tres equipos agrícolas. Results of normality tests for the three agricultural equipments.

\begin{tabular}{cccc}
\hline \multirow{2}{*}{ Equipo Agrícola } & \multicolumn{3}{c}{ Prueba de normalidad } \\
\cline { 2 - 4 } & Kolmogorov-Smirnov & Lilliefors & Shapiro-Wilk \\
\hline Arado de discos & $\mathrm{p}>0,20$ (ns) & $\mathrm{p}>0,20$ (ns) & $\mathrm{p}=0,87$ (ns) \\
Escarificador de muelles & $\mathrm{p}>0,20$ (ns) & $\mathrm{p}>0,20(\mathrm{~ns})$ & $\mathrm{p}=0,52$ (ns) \\
Sembradora-abonadora & $\mathrm{p}>0,20$ (ns) & $\mathrm{p}>0,20$ (ns) & $\mathrm{p}=0,54$ (ns) \\
\hline
\end{tabular}


En la Figura 6 se presentan los gráficos de residuos estandarizados para cada equipo agrícola, en función de la resistencia mecánica media del suelo a la penetración. De acuerdo con esos gráficos se deduce que no hay relación entre los residuos y las variables de los modelos elaborados, pues los puntos se sitúan aleatoriamente a lo largo del eje horizontal y no existen evidencias de algún padrón de dependencia funcional. Esa distribución se considera por MONTGOMERY et al. (2004), LEVINE et al. (2005) y WEISBERG (2005) como condición ideal en la elaboración de modelos empíricos.
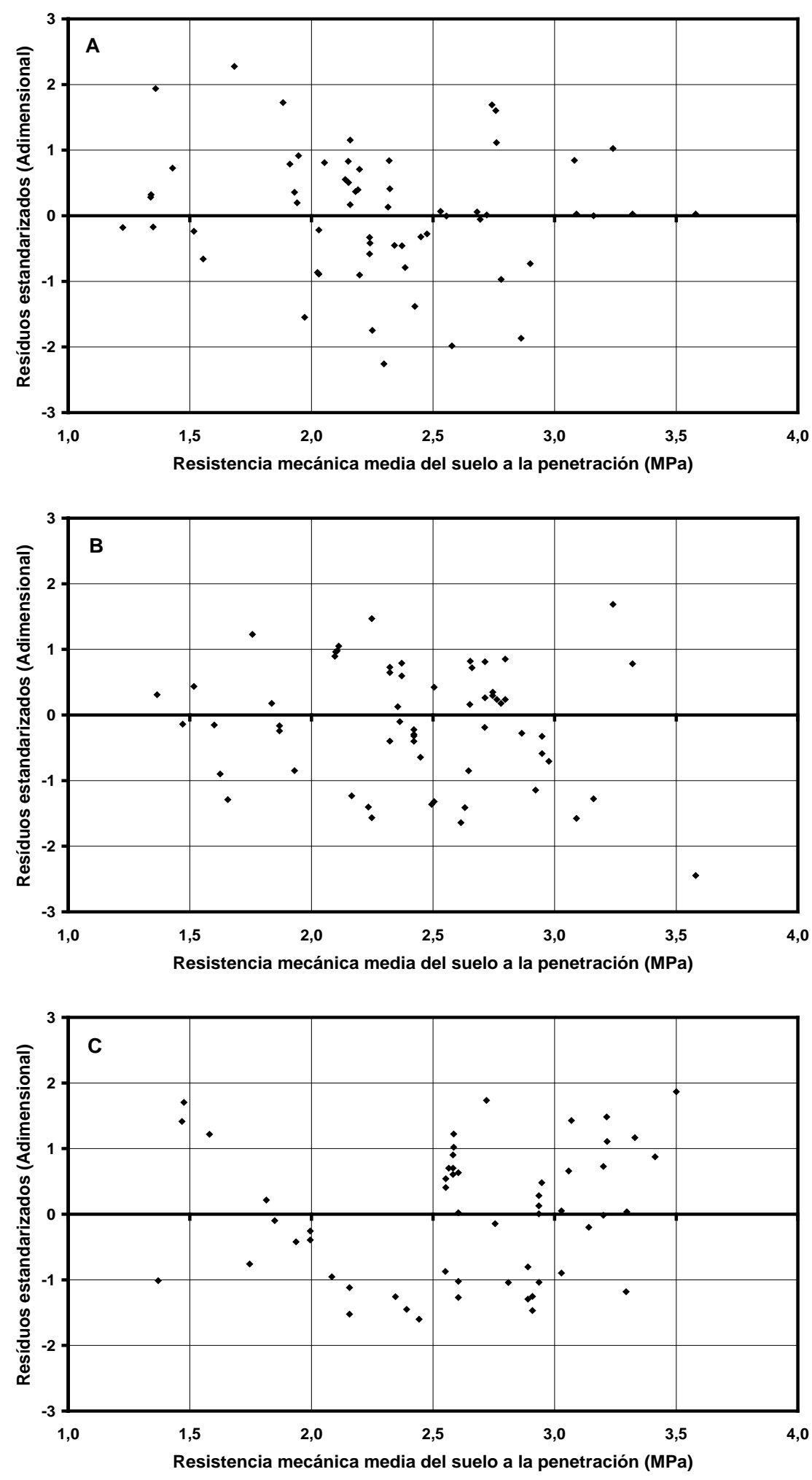

FIGURA 6. Residuos estandarizados en función de la resistencia mecánica media del suelo: a) arado de discos; b) escarificador; c) sembradora abonadora. Standardized residuals as function of mean soil mechanical resistance to penetration: a) disk plow; b) springs harrow; c) fertilizer seeder. 
En la Figura 6A se puede verificar que tres puntos se encuentran fuera del intervalo de residuos estandarizados de -2 a 2 . Sin embargo, esos puntos no superan el $5 \%$ del total y por tanto son observaciones atípicas que no alteran significativamente el ajuste de la ecuación (Figura 6). Para el caso del escarificador, se constata que solamente un punto se encuentra fuera de los límites del 95\% de confianza, teniendo poca importancia para la validación del modelo (Figura 6B). La Figura 6C indica sobreestimación de la fuerza, en función de la resistencia mecánica media del suelo a la penetración entre 2,0 y 2,5 MPa. Más, como son pocos valores y un pequeño intervalo, considerase que no afecta la eficiencia en la predicción.

Para confirmar analíticamente la adecuación de los modelos ajustados, los valores teóricos y experimentales de la fuerza de tracción fueron comparados estadísticamente, aplicándose la prueba t de Student. Los resultados obtenidos muestran que no hubo diferencias significativas entre los referidos valores (Tabla 4). Luego, se puede afirmar que las ecuaciones desarrolladas presentan adecuada confiabilidad, justificándose su aplicación, pues son apropiadas para determinar la fuerza de tracción requerida por los respectivos equipos agrícolas, en función de la resistencia mecánica media del suelo a la penetración.

Tabla 4. Resultados de la prueba t-Student para los tres equipos agrícolas. Results of the t-Student test for the three agricultural equipments.

\begin{tabular}{ccccc}
\hline \multirow{2}{*}{ Equipo Agrícola } & \multicolumn{3}{c}{ Valores de t } & \multirow{2}{*}{ Significancia } \\
\cline { 2 - 4 } & \multirow{2}{*}{ Calculado } & \multicolumn{2}{c}{ Tabulado } & \\
& & $\alpha=5 \%$ & \multirow{2}{*}{2,6479} & $\mathrm{~ns}$ \\
Arado de discos & 0,1566 & & $\mathrm{~ns}$ \\
Escarificador de muelles & 0,0332 & 1,9944 & $\mathrm{~ns}$ \\
Sembradora-abonadora & 0,3632 & & & $\mathrm{~ns}$ \\
\hline
\end{tabular}

Obs. $\alpha$ : Nivel de probabilidad; ns: no significativo

\section{CONCLUSIONES}

Las funciones exponenciales asociadas fueron apropiadas para estimar la fuerza de tracción requerida por equipos agrícolas, considerándose la resistencia mecánica del suelo a la penetración.

Las dependencias funcionales desarrolladas presentaron adecuada confiabilidad en las predicciones, lo que justifica su aplicación.

Los modelos obtenidos pueden ser una herramienta útil para el planeamiento y gestión de operaciones agrícolas mecanizadas en suelos con textura similar a las de esta investigación.

\section{REFERENCIAS}

ACOSTA, J. J. B. Índice de cone, capacidade de suporte de carga e teor de água de quatro solos da fazenda Lageado, Botucatu/SP: correlações e mapas de isocompactação. 2008. 145 f. Tese (Doutorado em Agronomia) - Faculdade de Ciências Agronômicas, Universidade Estadual Paulista, Botucatu, 2008.

ALMEIDA, R. A. S.; TAVARES-SILVA, C. A.; SILVA, S. L. Desempenho energético de um conjunto trator-semadora em função do escalonamento de marchas e rotações do motor. Revista Agrarian, Dourados, v.3, n.7, p.63-70, 2010.

ASAE. American Society of Agricultural Engineers. Agricultural machinery management data. In: ASAE Standards. St. Joseph, 2003. p.373-380. (ASAE D-497.4).

ASSIS, R. L.; LANÇAS, K. P. Avaliação dos atributos físicos de um nitossolo vermelho distroférrico sob sistema plantio direto, preparo convencional e mata nativa. Revista Brasileira de Ciência do Solo, Viçosa - MG, v.29, n.4, p.515-522, 2005. 
ASSIS, R. L.; LAZARINI, G. D.; LANÇAS, K. P.; CARGNELUTTI FILHO, A. Avaliação da resistência do solo à penetração em diferentes solos com a variação do teor de água. Engenharia Agrícola, Jaboticabal, v.29, n.4, p.558-568, out./dez. 2009.

BEUTLER, A. N.; CENTURION, J. F. Compactação do solo no desenvolvimento radicular e na produtividade de soja. Pesquisa Agropecuária Brasileira, Brasília, v.39, n.6, p.581-8, 2004.

BEUTLER, A. N.; CENTURION, J. F.; SILVA, A. P.; BARBOSA, J. C. Intervalo hídrico ótimo e produtividade de cultivares de soja. Revista Brasileira de Engenharia Agrícola \& Ambiental, Campina Grande, v.10, n.3, p.639-645, 2006.

BONNIN, J. J.; MIRÁS-ÁVALOS, J. M.; LANÇAS, K. P.; GONZÁLEZ, A. P.; VIEIRA, S. R. Spatial variability of soil resistance influenced by season of sampling. Bragantia, Campinas, v.69, Suplemento, p.163-173, 2010.

CARVALHO, G. J.; CARVALHO, M. P.; FREDDI, O. S.; MARTINS, M. V. Correlação da produtividade do feijão com a resistência à penetração do solo sob plantio direto. Revista Brasileira de Engenharia Agrícola \& Ambiental, Campina Grande, v.10, n.3, p.765-771, 2006.

CUNHA, J. P. A. R.; CARVALHO JÚNIOR, P. C.; SOUZA, J. V.; BORGES, E. N.; REIS, E. F. Compactação do solo sob sistemas de manejo convencionais e conservacionistas. Revista Engenharia na Agricultura, Viçosa - MG, v.17, n.2, p. 155-162, 2009.

EMBRAPA. Empresa Brasileira de Pesquisa Agropecuária. Centro Nacional de Pesquisa dos solos. Sistema brasileiro de classificação dos solos. Brasília, 1999. 412 p.

FERNANDES, J. C.; GAMERO, C. A. Avaliação do desempenho das máquinas agrícolas na implantação da cultura do girassol. Revista Energia na Agricultura, Botucatu, v.25, n.2, p.74-87, 2010.

FURLANI, C. E. A.; GAMERO, C. A.; LEVIEN, R.; LOPES, A. Desempenho operacional de uma semeadora-adubadora de precisão, em função do preparo do solo e do manejo de cobertura de inverno. Engenharia Agrícola, Jaboticabal, v.24, n.2, p.388-395, maio/ago. 2004.

FURLANI, C. E. A.; PAVAN JÚNIOR, A.; LOPES, A.; SILVA, R. P.; GROTTA, D. C. C.; CORTEZ, J. W. Desempenho operacional de semeadora-adubadora em diferentes manejos da cobertura e da velocidade. Engenharia Agrícola, Jaboticabal, v. 27, n. 2, p.456-462, maio/ago, 2007.

KÖEPPEN, W.; GEIGER, R. Klima der Erde. Gotha: Verlag Justus Perthes. 1928.

LEVIEN, R.; MARQUES, J.P.; BENEZ, S.H. Desempenho de uma semeadora-adubadora de precisão, em semeadura de milho (Zea mays L.), sob diferentes formas de manejo do solo. In: CONGRESSO BRASILEIRO DE ENGENHARIA AGRÍCOLA, 28. 1999, Pelotas. Anais... Pelotas: Sociedade Brasileira de Engenharia Agrícola, 1999. 1 CD-ROM.

LEVINE, D.M.; STEPHAN, D.; KREHBIEL, T.C.; BERENSON, M.L. Estatística - Teoria e aplicações usando-se o Microsoft EXCEL em português. Rio de Janeiro: Livros Técnicos e Científicos Editora, 2005. 819 p.

MACHADO, R. L. T.; FERREIRA, M. F.; MACHADO, A. L. T.; REIS, A. V.; BUTZKE, H. P. Força de tração de semeadoras para plantio direto: informações de campo x informações de catálogo. Tecno-Lógica, Santa Cruz do Sul, v.11, n.1 e 2, p.15-17, jan./dez. 2007.

MELLO, R. C.; MAGALHÃES, P. S. G. Desempenho operacional de um arado de discos. Bragantia, Campinas, v.54, n.2, p.447-454, 1995.

MERCANTE, E.; SILVA, S. L.; MODOLO, A. J.; SILVEIRA, J. C. M. Demanda energética e distribuição de sementes de milho em função da velocidade de duas semeadoras. Revista Brasileira de Engenharia Agrícola \& Ambiental, Campina Grande, v.9, n.3, p.424-428, 2005. 
MODOLO, A. J.; SILVA, S. L.; SILVEIRA, J. C. M.; MERCANTE, E. Avaliação do desempenho de duas semeadoras-adubadoras de precisão em diferentes velocidades. Engenharia na Agricultura, Viçosa, MG, v.12, n.4, p.298-306, out./dez. 2004.

MODOLO, A. J.; GABRIEL FILHO, A.; SILVA, S. L.; GNOATTO, E. Força de tração necessária em função do número de linhas de semeadura utilizadas por uma semeadora-adubadora de precisão. Engenharia Agrícola, Jaboticabal, v. 25, n. 2, p.465-473, maio/ago. 2005.

MOLIN, J. P.; MAGALHÃES, R. P.; FAULIN, G. D. Análise espacial da ocorrência do índice de cone em área sob semeadura direta e sua relação com fatores do solo. Engenharia Agrícola, Jaboticabal, v. 26, n. 2, p.442-452, maio/ago. 2006.

MONTGOMERY, D. C.; RUNGER, G. C.; HUBELE, N. F. Estatística aplicada à engenharia. Rio de Janeiro: LTC, 2004. 335 p.

PÉREZ, M. Rendimiento de un tractor agrícola en función del sistema de labranza y la carga. I. Características de la tracción. Revista Bioagro, Barquisimeto, v.18, n.1, p.49-55, 2006.

SALVADOR, N.; BENEZ, S. H.; MION, R. L. Consumo de combustível na operação de subsolagem realizada antes e depois de diferentes sistemas de preparo periódico do solo. Engenharia Agrícola, Jaboticabal, v.28, n.2, p.256-262, abr./jun., 2008.

SANTOS, P. M. Modelagem do desempenho em tração de conjuntos mecanizados visando ao dimensionamento do trator. 2010. 162 f. Tese (Doutorado em Engenharia Agrícola) - Universidade Federal de Santa Maria, Centro de Ciências Rurais, Santa Maria, 2010.

SILVEIRA, J. C. M.; GABRIEL FILHO, A.; SECCO, D. Demanda de potência e força de tração de uma semeadora na implantação do milho safrinha sob plantio direto. Engenharia na Agricultura, Viçosa, v.13, n.4, p. 256-267, Out./Dez., 2005 a.

SILVEIRA, J. C. M.; MODOLO, A. J.; SILVA, S. L.; GABRIEL FILHO, A. Força de tração e potência de uma semeadora em duas velocidades de deslocamento e duas profundidades de deposição de sementes. Revista Brasileira de Engenharia Agrícola \& Ambiental, Campina Grande, v.9, n.1, p.125-128, 2005 b.

SILVEIRA, J. C. M.; FERNANDES, H. C.; MODOLO, A. J.; SILVA, S. L.; TROGELLO, E. Furrow depth, soil disturbance area and draft force of a seeder-fertilizer at different seeding speeds. Revista Ceres, Viçosa - MG, v.58, n.3, p.293-298, 2011.

VENIALGO, C. A.; INGARAMO, O.; IBALO, S.; ROLDÁN, M. F.; BANZHAF, G.;

GUTIERREZ, N. Índice de cono, humedad presente y densidad aparente en diferentes labranzas y rotaciones. Corrientes: Comunicaciones Científicas y Tecnológicas, Universidad Nacional del Nordeste de Argentina, A-076, 2004.

WEIRICH NETO, P. H.; BORGHI, E.; SVERZUT, C. B.; MANTOVANI, E. C.; GOMIDE, R. L.; NEWES, W. L. C. Análise multivariada da resistência do solo à penetração sob plantio direto. Ciência Rural, Santa Maria, v.36, n.4, p.1186-1192, jul-ago, 2006.

WEISBERG, S. Applied linear regression. New York: John Wiley \& Sons, 2005. 330 p. 